\title{
Accuracy of X-Ray Image-Based 3D Localization from Two C-Arm Views: A Comparison Between an Ideal System and a Real Device
}

\author{
Alexander Brost ${ }^{a}$, Norbert Strobel ${ }^{b}$, Liron Yatziv ${ }^{c}$, Wesley Gilson ${ }^{d}$, Bernhard Meyer ${ }^{e}$, Joachim \\ Hornegger ${ }^{a}$, Jonathan Lewin ${ }^{f}$ and Frank Wacker $f$ \\ ${ }^{a}$ Chair of Pattern Recognition, Department of Computer Science, \\ Friedrich-Alexander-University Erlangen-Nuremberg, Erlangen, Germany; \\ ${ }^{b}$ Klinik und Hochschulambulanz für Radiologie und Nuklearmedizin, Charité \\ Universitätsmedizin Berlin, Campus Benjamin Franklin, Berlin, Germany; \\ ${ }^{c}$ Siemens Corporate Research, Princeton, NJ, USA; \\ ${ }^{d}$ Siemens Corporate Research, Baltimore, MD, USA; \\ ${ }^{e}$ Siemens AG, Healthcare Sector, Forchheim, Germany;

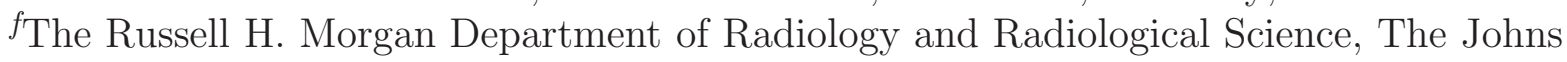 \\ Hopkins University School of Medicine, Baltimore, MD, USA
}

\begin{abstract}
C-arm X-ray imaging devices are commonly used for minimally invasive cardiovascular or other interventional procedures. Calibrated state-of-the-art systems can, however, not only be used for 2D imaging but also for three-dimensional reconstruction either using tomographic techniques or even stereotactic approaches.

To evaluate the accuracy of X-ray object localization from two views, a simulation study assuming an ideal imaging geometry was carried out first. This was backed up with a phantom experiment involving a real C-arm angiography system. Both studies were based on a phantom comprising five point objects. These point objects were projected onto a flat-panel detector under different $\mathrm{C}$-arm view positions. The resulting $2 \mathrm{D}$ positions were perturbed by adding Gaussian noise to simulate 2D point localization errors. In the next step, 3D point positions were triangulated from two views. A 3D error was computed by taking differences between the reconstructed 3D positions using the perturbed 2D positions and the initial 3D positions of the five points. This experiment was repeated for various C-arm angulations involving angular differences ranging from $15^{\circ}$ to $165^{\circ}$. The smallest 3D reconstruction error was achieved, as expected, by views that were $90^{\circ}$ degrees apart. In this case, the simulation study yielded a 3D error of $0.82 \mathrm{~mm} \pm 0.24 \mathrm{~mm}$ (mean \pm standard deviation) for 2D noise with a standard deviation of $1.232 \mathrm{~mm}$ (4 detector pixels). The experimental result for this view configuration obtained on an AXIOM Artis C-arm (Siemens AG, Healthcare Sector, Forchheim, Germany) system was $0.98 \mathrm{~mm} \pm 0.29 \mathrm{~mm}$, respectively.

These results show that state-of-the-art C-arm systems can localize instruments with millimeter accuracy, and that they can accomplish this almost as well as an idealized theoretical counterpart. High stereotactic localization accuracy, good patient access, and CT-like 3D imaging capabilities render state-of-the-art C-arm systems ideal devices for X-ray based minimally invasive procedures.
\end{abstract}

Keywords: accuracy, c-arm, localization, projection, reconstruction, triangulation

\section{INTRODUCTION}

C-arm systems are devices to perform local therapy using fluoroscopic X-ray imaging. State-of-the-art systems are hybrid devices offering 2D and 3D imaging capabilities ${ }^{1}$. Multi-modality image integration is possible by registering $3 \mathrm{D}$ data sets generated from other sources to the $\mathrm{C}$-arm device ${ }^{2,3}$. If the $\mathrm{C}$-arm projection geometry

Send correspondence to Alexander Brost: E-mail: Alexander.Brost@gmx.de 
is known, e.g., by calibration, it is possible to generate $2 \mathrm{D}$ renderings from the $3 \mathrm{D}$ data sets. Those images can be combined with live fluoro scenes for image-based navigation and guidance ${ }^{4}$. A commercially available product implementing this approach is syngo iPilot (Siemens AG, Healthcare Sector, Forchheim, Germany). A calibrated C-arm system can, however, not only perform 3D tomographic cone-beam reconstruction, but it can also be used to reconstruct a 3D point using stereotactic techniques. While used for the placement of electrodes to known physical locations for the mapping of the brains electrical activity and functional response ${ }^{5}$, the most common clinical application for the stereotactic approach probably is breast biopsy ${ }^{6}$. In this case, the 3D position of a target is recovered by identifying its position on mammograms taken from two different directions.

An interesting question is how accurate state-of-the-art interventional C-arm systems are when used for localization of a 3D point from two X-ray views. To find an answer, we introduce C-arm projection geometry and projection matrices first. Among others, they can be used to compute 3D points from their corresponding $2 \mathrm{D}$ shadows acquired under different viewing angles. Afterwards we turn to the $3 \mathrm{D}$ reconstruction error. It is our figure of merit. In the next step we explain our setup for simulation study and experiment. Finally we present our results and discuss them.

\section{METHODS}

For 3D point-reconstruction from two views ${ }^{7}$, projection matrices are used. These matrices can be obtained either by calculation assuming an ideal geometric model of the $\mathrm{C}$-arm, or by calibration, taking into account the non-ideal behavior of the C-arm system's projection geometry. The calibration of camera systems has been thoroughly studied, and well established techniques exist ${ }^{8,9}$.

\subsection{Acquisition Geometry and Projection Matrices}

The viewing orientation of a C-arm system is characterized by a primary and secondary anatomic view angle. The primary angle (RAO/LAO) reports how much a C-arm has been rotated to a patient's right/left side (right anterior oblique/left anterior oblique). The secondary angle (CRAN/CAUD) tells how much a C-arm has been angulated toward a patient's head (cranial) or feet (caudal) direction. In addition, it is possible to change the source-to-detector distance (SID) of the system by moving the detector closer to or further away from the patient.

It is possible to model the C-arm X-ray imaging system as a pinhole camera. In this case, a projection matrix can be used to describe how a $3 \mathrm{D}$ point is mapped to its corresponding shadow in the projection image. A projection matrix $\mathbf{P} \in \mathbb{R}^{3 \times 4}$ is defined as ${ }^{7,8,10}$

$$
\mathbf{P}=\mathbf{K}[\mathbf{R} \mid \mathbf{t}]
$$

Here, $\mathbf{K} \in \mathbb{R}^{3 \times 3}$ represents the intrinsic camera parameters. The matrix $\mathbf{R} \in \mathbb{R}^{3 \times 3}$ and the vector $\mathbf{t} \in \mathbb{R}^{3}$ comprise the extrinsic camera parameters for rotation and translation, respectively. Taking some change in image coordinate system into account, we can express the intrinsic camera parameter matrix as

$$
\mathbf{K}=\left(\begin{array}{ccc}
\frac{\mathrm{SID}}{p} & 0 & o_{x} \\
0 & \frac{\mathrm{SID}}{p} & o_{y} \\
0 & 0 & 1
\end{array}\right) \cdot\left(\begin{array}{ccc}
1 & 0 & 0 \\
0 & 0 & -1 \\
0 & 1 & 0
\end{array}\right)
$$

The SID is defined as the source-to-image-distance, and $p$ is the pixel size (assumed to be identical in $x$ and $y$ directions). The parameters $o_{x}$ and $o_{y}$ are the coordinates of the isocenter in the image plane. The translational part of the extrinsic parameters can be defined as

$$
\mathbf{t}=\left(\begin{array}{c}
0 \\
\mathrm{SOD} \\
0
\end{array}\right)
$$

The origin of the coordinate system coincides with the isocenter of the C-arm. The variable SOD is the sourceto-object-distance. It is identical to the distance from the X-ray focus to the iso-center of the $\mathrm{C}$-arm system. We also have

$$
\mathrm{SID}=\mathrm{SOD}+\mathrm{OID}
$$




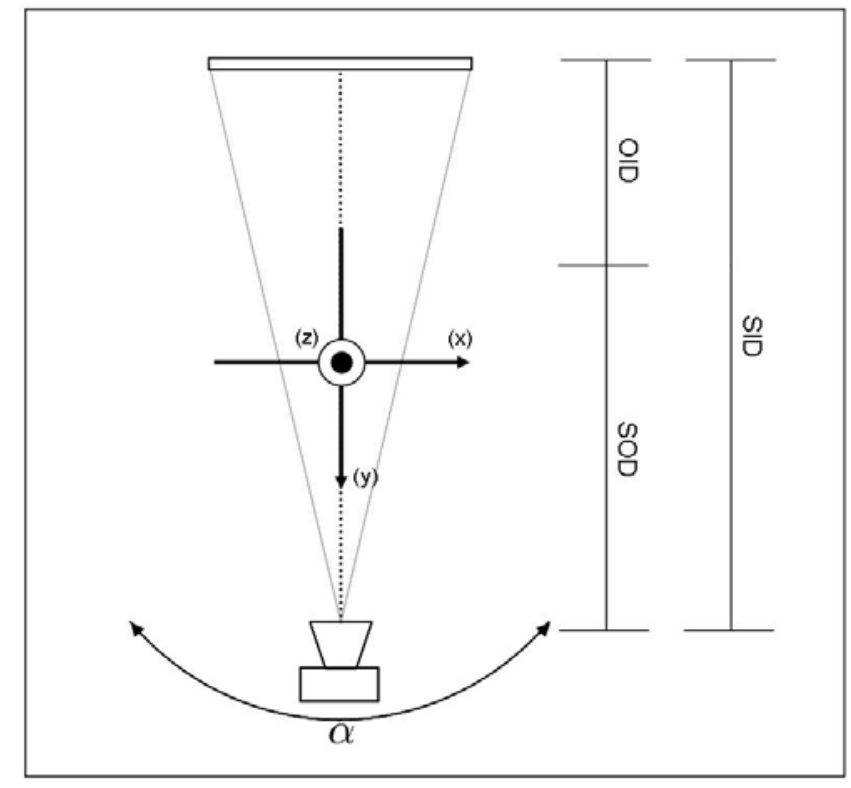

Figure 1. Basic projection view geometry of a C-arm X-ray imaging system: SID denotes the source-to-image-distance, SOD the source-to-object-distance and OID the object-to-image distance. The iso-center is the origin of the world coordinate system. The $z$-axis points towards the reader.

where OID is the object-to-image-distance. These parameters are illustrated in Fig. 1. For this study, we focus on the primary angle only. The secondary angle was not considered, i.e., the CRAN/CAUD view angle was set to zero. In this case, we can write the rotation matrix $\mathbf{R}$ as

$$
\mathbf{R}=\left(\begin{array}{ccc}
\cos (\alpha) & -\sin (\alpha) & 0 \\
\sin (\alpha) & \cos (\alpha) & 0 \\
0 & 0 & 1
\end{array}\right)
$$

where $\alpha$ is the angle in $\mathrm{RAO} / \mathrm{LAO}$ view direction.

\subsection{Algorithm for 3D Reconstruction from two Views}

The algorithm used for reconstructing points in 3D from two 2D projections is taken from Schmidt ${ }^{7}$. We recall Eq. (1)

$$
\mathbf{P}=\mathbf{K}[\mathbf{R} \mid \mathbf{t}]
$$

Since we need two projections for reconstruction, we use the subscript $\alpha$ for the projection matrices, highlighting the angle of the C-arm for the projection. This notation results in $\mathbf{P}_{\alpha}=\mathbf{K}_{\alpha}\left[\mathbf{R}_{\alpha} \mid \mathbf{t}_{\alpha}\right]$, where $\mathbf{K}_{\alpha}, \mathbf{R}_{\alpha} \in \mathbb{R}^{3 \times 3}$, $\mathbf{t}_{\alpha} \in \mathbb{R}^{3}$. Given a world point in $3 \mathrm{D}, \mathbf{w} \in \mathbb{R}^{3}$, its projection can be calculated as

$$
\tilde{\mathbf{v}}_{\alpha}=\mathbf{P}_{\alpha} \cdot \tilde{\mathbf{w}}
$$

where $\tilde{\mathbf{v}}_{\alpha} \in \mathbb{R}^{3}$ represents the resulting $2 \mathrm{D}$ point in the image plane in homogeneous coordinates and $\tilde{\mathbf{w}}=$ $\left(\mathbf{w}^{T}, 1\right)^{T}$ is the world point also expressed in homogeneous coordinates. Using the fact that the fourth component of the vector $\tilde{\mathbf{w}}$ is 1 and that a projection matrix can be expressed as shown in Eq. (6), we get

$$
\tilde{\mathbf{v}}_{\alpha}=\mathbf{K}_{\alpha}\left(\mathbf{R}_{\alpha} \cdot \mathbf{w}+\mathbf{t}_{\alpha}\right) \text {. }
$$

Solving for the world point $\mathbf{w}$ in non-homogeneous coordinates, we get

$$
\mathbf{w}=\mathbf{R}_{\alpha}^{-1} \cdot\left(\mathbf{K}_{\alpha}^{-1} \cdot \tilde{\mathbf{v}}_{\alpha}-\mathbf{t}_{\alpha}\right) .
$$


Since the image point $\tilde{\mathbf{v}}_{\alpha}$ is given in homogeneous coordinates, the exact coordinates of the points are only known up to a scaling factor $\lambda \in \mathbb{R}$. Hence, we can write

$$
\tilde{\mathbf{v}}_{\alpha}=\left(\begin{array}{c}
\lambda u_{\alpha} \\
\lambda v_{\alpha} \\
\lambda
\end{array}\right)=\left(\begin{array}{c}
\lambda \mathbf{v}_{\alpha} \\
\lambda
\end{array}\right)=\lambda\left(\begin{array}{c}
\mathbf{v}_{\alpha} \\
1
\end{array}\right)=\lambda \tilde{\mathbf{v}}_{\alpha}^{\star}
$$

with $\tilde{\mathbf{v}}_{\alpha}^{\star}=\left(\mathbf{v}_{\alpha}, 1\right)^{T}$ and $\mathbf{v}_{\alpha}=\left(u_{\alpha}, v_{\alpha}\right)^{T}$ representing the actual 2D image coordinates at the detector for a view angle $\alpha$. This fact can be used to construct a line through the 2D image point and the world point. A point along this line can be described as

$$
\begin{aligned}
\mathbf{r}_{\alpha, \lambda} & =\mathbf{R}_{\alpha}^{-1} \cdot\left(\mathbf{K}_{\alpha}^{-1} \cdot \lambda \cdot \tilde{\mathbf{v}}_{\alpha}^{\star}-\mathbf{t}_{\alpha}\right) \\
& =\lambda \cdot \mathbf{R}_{\alpha}^{-1} \mathbf{K}_{\alpha}^{-1} \tilde{\mathbf{v}}_{\alpha}^{\star}-\mathbf{R}_{\alpha}^{-1} \cdot \mathbf{t}_{\alpha} .
\end{aligned}
$$

Substituting the point of origin $\mathbf{o}_{\alpha}=-\mathbf{R}_{\alpha}^{-1} \cdot \mathbf{t}_{\alpha}$ and the direction $\mathbf{d}_{\alpha}=\mathbf{R}_{\alpha}^{-1} \mathbf{K}_{\alpha}^{-1} \tilde{\mathbf{v}}_{\alpha}^{\star}$, Eq. (11) becomes

$$
\mathbf{r}_{\alpha, \lambda}=\mathbf{o}_{\alpha}+\lambda \cdot \mathbf{d}_{\alpha}
$$

As both lines are assumed to pass through the same world point, we can write

$$
\begin{aligned}
& \mathbf{w}=\mathbf{o}_{\alpha_{1}}+\nu \mathbf{d}_{\alpha_{1}} \\
& \mathbf{w}=\mathbf{o}_{\alpha_{2}}+\mu \mathbf{d}_{\alpha_{2}}
\end{aligned}
$$

with $\nu, \mu \in \mathbb{R}$. Note that $\alpha_{1}$ and $\alpha_{2}$ are different values for $\alpha$ representing two C-arm viewing angles. Due to errors during the selection of points in $2 \mathrm{D}$ projections associated with the same $3 \mathrm{D}$ object, the lines do not necessarily intersect. We rearrange terms and get

$$
\mathbf{o}_{\alpha_{2}}-\mathbf{o}_{\alpha_{1}}=\nu \mathbf{d}_{\alpha_{1}}-\mu \mathbf{d}_{\alpha_{2}} .
$$

This equation can be solved in a least squares approach for $\mu$ and $\nu$. To this end, it is beneficial to reformulate Eq. (15) as

$$
\mathbf{o}_{\alpha_{2}}-\mathbf{o}_{\alpha_{1}}=\left[\mathbf{d}_{\alpha_{1}}, \mathbf{d}_{\alpha_{2}}\right] \cdot\left(\begin{array}{c}
\nu \\
-\mu
\end{array}\right) \text {. }
$$

Using the substitutions

$$
\mathbf{b}=\mathbf{o}_{\alpha_{2}}-\mathbf{o}_{\alpha_{1}}
$$

with $\mathbf{b} \in \mathbb{R}^{3}$ and

$$
\mathbf{C}=\left[\mathbf{d}_{\alpha_{1}}, \mathbf{d}_{\alpha_{2}}\right]
$$

with $\mathbf{C} \in \mathbb{R}^{3 \times 2}$, Eq. (16) can be rewritten as

$$
\mathbf{b}-\mathbf{C} \cdot\left(\begin{array}{c}
\nu \\
-\mu
\end{array}\right)=0 .
$$

The unknowns $\nu$ and $\mu$ can be found using a least squares approach like the pseudo-inverse $\mathbf{C}^{\dagger}$ which is defined as $^{11}$

$$
\mathbf{C}^{\dagger}=\left(\mathbf{C}^{T} \cdot \mathbf{C}\right)^{-1} \cdot \mathbf{C}^{T}
$$

Applying this to Eq. (19), the unknowns can be found by

$$
\left(\begin{array}{c}
\nu \\
-\mu
\end{array}\right)=\mathbf{C}^{\dagger} \mathbf{b}
$$

Finally, the point in 3D, reconstructed from two views, characterized by $\alpha_{1}$ and $\alpha_{2}$, is calculated as the averaged sum of Eqs. (13) and (14). We get

$$
\mathbf{w}_{\alpha_{1}, \alpha_{2}}^{\star}=\frac{1}{2} \cdot\left(\mathbf{o}_{\alpha_{1}}+\nu \mathbf{d}_{\alpha_{1}}+\mathbf{o}_{\alpha_{2}}+\mu \mathbf{d}_{\alpha_{2}}\right) .
$$




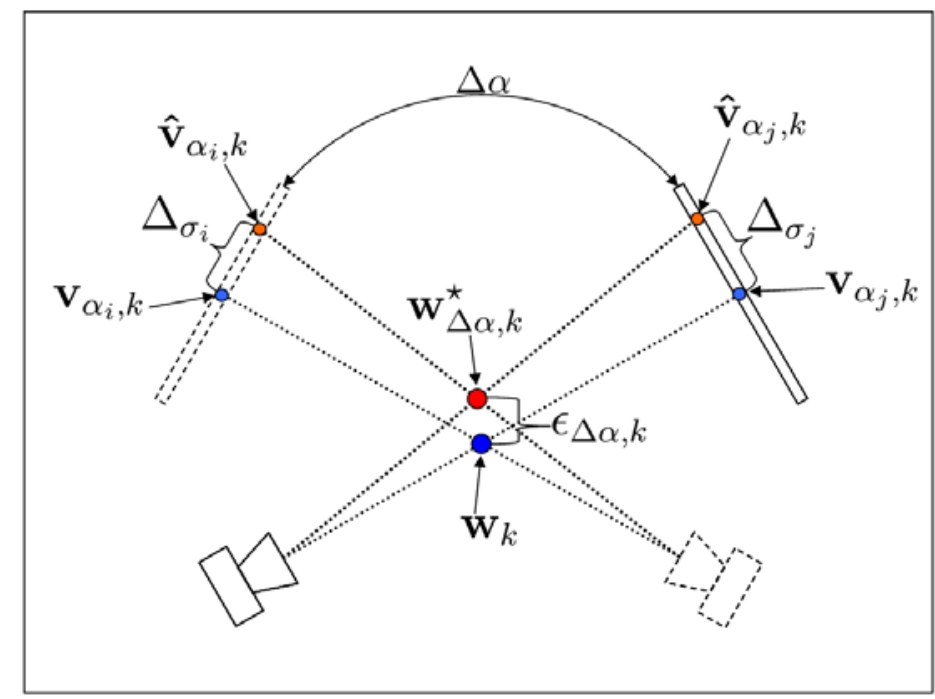

Figure 2. The relative angle between to projections is defined as their angular difference $\Delta \alpha$. The 3D reconstruction error for a particular view difference $\Delta \alpha$, measured for a certain $3 \mathrm{D}$ point $\mathbf{w}_{k}$ (blue) and a specific perturbation $i$ is called $\epsilon_{\Delta \alpha, k}$. The reconstructed point from noisy data is $\hat{\mathbf{w}}_{\Delta \alpha, k}^{\star}(\mathrm{red})$.

To indicate that there are multiple $3 \mathrm{D}$ points (five in our case), we add the subscript $k$ to $\mathbf{w}_{\alpha_{1}, \alpha_{2}}^{\star}$. That is, the $k$-th reconstructed point is $\mathbf{w}_{\alpha_{1}, \alpha_{2}, k}^{\star}$ with $k=1, \ldots, 5$. The $3 \mathrm{D}$ reconstruction at a particular point $\mathbf{w}_{k}$ can be summarized as

$$
\mathbf{w}_{\alpha_{1}, \alpha_{2}, k}^{\star}=\operatorname{recon}\left(\mathbf{v}_{\alpha_{1}, k}, \mathbf{P}_{\alpha_{1}}, \mathbf{v}_{\alpha_{2}, k}, \mathbf{P}_{\alpha_{2}}\right)
$$

with $\mathbf{v}_{\alpha_{i}, k}$ being the projected image point of $\mathbf{w}_{k}$ under projection angle $\alpha_{i}$. To introduce errors in the $2 \mathrm{D}$ localization step, the 2D points are disturbed by Gaussian noise. The perturbed image points are denoted as:

$$
\hat{\mathbf{v}}_{\alpha_{i}, k}=\mathbf{v}_{\alpha_{i}, k}+\Delta_{\sigma_{i}}
$$

with $\Delta_{\sigma_{i}}\left(\Delta_{\sigma_{j}}\right)$ being a two-dimensional perturbation vector assumed to be Gaussian distributed with standard deviation $\sigma$. It is added to the 2D point, $\mathbf{v}_{\alpha_{i}, k}\left(\mathbf{v}_{\alpha_{j}, k}\right)$, in one of eight directions. Using perturbed 2D positions $\hat{\mathbf{v}}_{\alpha_{i}, k}$, Eq. (23) becomes

$$
\hat{\mathbf{w}}_{\alpha_{1}, \alpha_{2}, k}^{\star}=\operatorname{recon}\left(\hat{\mathbf{v}}_{\alpha_{1}, k}, \mathbf{P}_{\alpha_{1}}, \hat{\mathbf{v}}_{\alpha_{2}, k}, \mathbf{P}_{\alpha_{2}}\right)
$$

where $\hat{\mathbf{w}}_{\alpha_{1}, \alpha_{2}, k}^{\star}$ represents a set of reconstructed points computed after adding $\Delta_{\sigma_{i}}\left(\Delta_{\sigma_{j}}\right)$ to the two points in one of $N=8$ directions. Since a 3D point is triangulated from two views, and because each 2D point is perturbed, we get a total of $N^{2}$ three-dimensional positions for each 3D point observed at a particular view combination defined by angles $\alpha_{1}$ and $\alpha_{2}$. To simplify our analysis, we did not consider absolute angles, but relative angles, i.e. we studied the reconstruction error with respect to

$$
\Delta \alpha=\left|\alpha_{1}-\alpha_{2}\right| .
$$

\subsection{Reconstruction Error}

To find the 3D reconstruction error, localization by triangulation was performed and the Euclidean norm between the reconstructed 3D position and the corresponding 3D point in the tomographic volume data set was calculated. This was performed for all five points of the phantom.

The $3 \mathrm{D}$ reconstruction error for a particular view difference $\Delta \alpha$, measured for a certain $3 \mathrm{D}$ point $\mathbf{w}_{k}$ is defined as

$$
\epsilon_{\Delta \alpha, k}=\left\|\mathbf{w}_{k}-\hat{\mathbf{w}}_{\Delta \alpha, k}^{\star}\right\|_{2}
$$


with $\hat{\mathbf{w}}_{\Delta \alpha, k}^{\star}$ being the reconstructed point from noisy data. For an illustration of view angle difference, $\Delta \alpha$ and the associated 3D reconstruction error, $\epsilon_{\Delta \alpha, k}$, see Fig. 2.

We consider the mean reconstruction error $\bar{\epsilon}_{\Delta_{\alpha}}$ for each relative angle and its standard deviation $\sigma_{\epsilon_{\Delta \alpha}}$ as our figure of merit. The mean reconstruction error is defined as

$$
\bar{\epsilon}_{\Delta \alpha}=\frac{1}{K * N^{2}} \sum_{k} \epsilon_{\Delta_{\alpha}, k}
$$

The standard deviation as of $\bar{\epsilon}_{\Delta \alpha}$ is

$$
\sigma_{\epsilon \Delta \alpha}=\sqrt{\frac{1}{K * N^{2}-1} \sum_{k}\left(\epsilon_{\Delta \alpha, k}-\bar{\epsilon}_{\Delta \alpha}\right)^{2}} .
$$

\section{MATERIALS}

Both our simulation and experiments revolved around a custom-made phantom comprising five point objects and an AXIOM Artis dFA C-arm device (Siemens AG, Healthcare Sector, Forchheim, Germany). The work was performed at the Johns Hopkins Hospital (Baltimore, MD, USA), see Fig. 3. The AXIOM Artis dFA facilitates 3D cone beam reconstruction carried out using syngo DynaCT (Siemens AG, Healthcare Sector, Forchheim, Germany $)^{12-15}$. The system has a flat-panel detector with a detector pixel size of $0.308 \mathrm{~mm}$. Its 3D spatial resolution is $0.25 \mathrm{~mm}^{1}$.

The 2D center positions of the 3D points' X-ray shadows were obtained by forward projecting the 3D positions derived from the $3 \mathrm{D}$ data set. The actual 2D points later used for triangulation were generated by perturbing the initial 2D positions, i.e., offsetting them from their original position by adding Gaussian noise with a standard deviation of $1.232 \mathrm{~mm}$ (four pixels) in each of eight possible directions.

\subsection{Phantom and C-arm Imaging}

The custom-made phantom comprised five point objects embedded in a box filled with gelatin of size $15 \mathrm{~cm} \mathrm{x}$ $10 \mathrm{~cm} \times 5 \mathrm{~cm}$. The radio-opaque sphere-shaped objects with a diameter of about $2 \mathrm{~mm}$ were arranged such that their shadows showed minimal overlap when taking X-ray projections from various angles.

A 3D dataset of the custom made phantom was used as ground truth both for the simulation study and also for the experimental verification. The center coordinates of the 3D point positions were determined. These center positions were forward projected either assuming an ideal (or real) C-arm imaging geometry to obtain $2 \mathrm{D}$ locations for the simulation study (or the experimental verification). The ideal projection geometry is characterized by a source-detector distance (SID) of $120 \mathrm{~cm}$ and a distance from source to iso-center (SOD) of $75 \mathrm{~cm}$. The pixel size at the detector was set to $0.308 \mathrm{~mm}$. For the actual C-arm experiment, online projection matrices were used. These projection matrices are output when the system is in operation. Based on a calibration run, they take system imperfections into account ${ }^{16}$. In either case, an ensemble of perturbed 3D positions was calculated by triangulation and evaluated using Eqs. (28) and (29), respectively.

For this report, we only selected $\mathrm{C}$-arm positions along $\mathrm{RAO} / \mathrm{LAO}$ view directions. In other words, 2D projections of the 3D positions were determined by varying the primary (LAO/RAO) angle only. No angulation in cranial or caudal direction was performed. The LAO/RAO viewing angles ranged from $-180^{\circ}$ to $180^{\circ}$ in steps of $15^{\circ}$. The view angle differences, $\Delta \alpha$, were taken from $15^{\circ}$ to $165^{\circ}$.

\subsection{D Point Reconstruction}

For every combination of two viewing angles, the corresponding projection matrices and perturbed 2D points were used for 3D reconstruction according to Section 2.2. An example of such an image pair is shown in Fig. 4. There a red circle indicates the $90 \%$ confidence interval around the ground truth. The ground truth in $2 \mathrm{D}$ is given by the forward projected 3D point position that was determined in the $3 \mathrm{D}$ volumetric data set. 


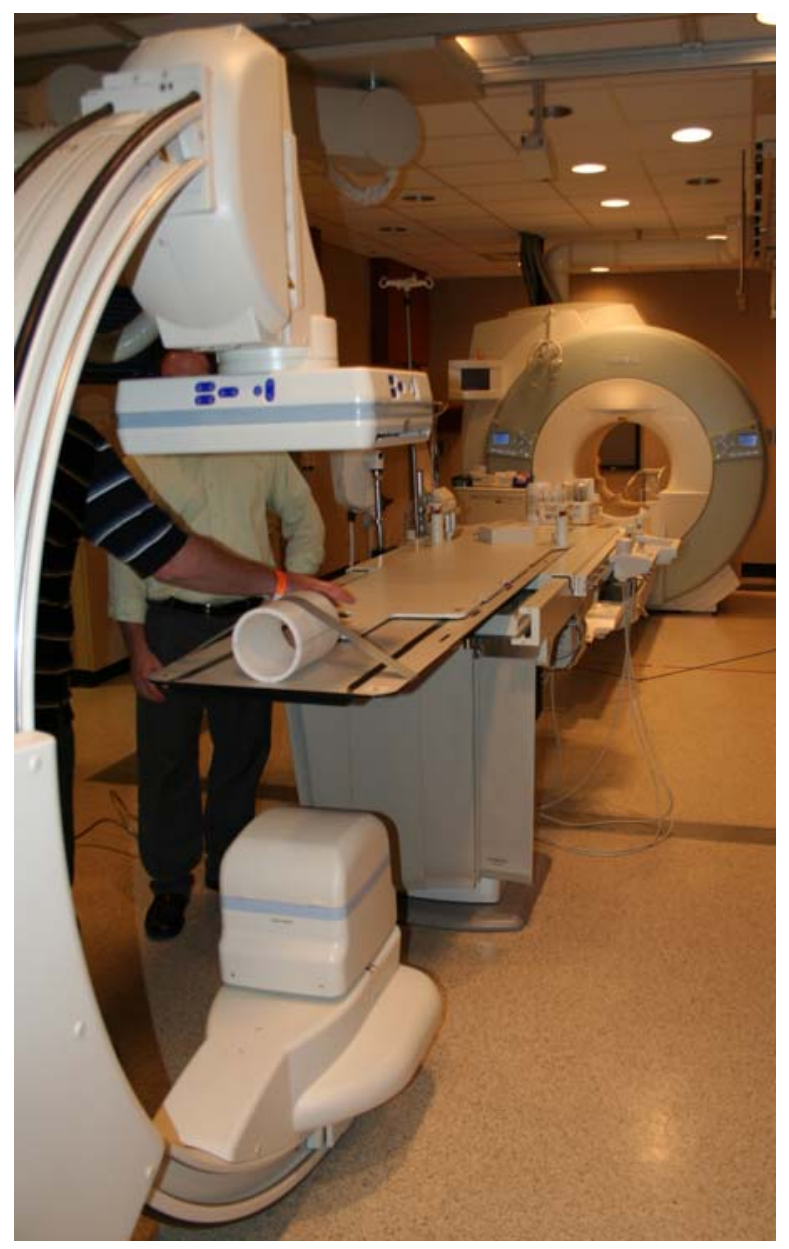

Figure 3. AXIOM Artis dFA C-arm imaging system (Siemens AG, Healthcare Sector, Forchheim, Germany) at the Johns Hopkins Hospital (Baltimore, MD, USA). It was used to generate 2D X-ray projections as well as a 3D data set of a custom-made phantom. C-arm 3D imaging was performed using syngo DynaCT (Siemens AG, Healthcare Sector, Forchheim, Germany).

\section{RESULTS}

After triangulation was performed for all possible view combinations, the 3D reconstruction error and the standard deviation for every angular view difference was calculated as described in Section 2.3. To arrive at a mean error for a particular angular view difference, all associated 3D errors are averaged as defined in Eq. (28). The standard deviation for each error was calculated using Eq. (29).

The results for the simulation study were obtained using projections matrices assuming an ideal C-arm imaging geometry. The experimental evaluation was based on online projection matrices generated by the system. These projection matrices take into account that the real projection geometry of an actual C-arm imaging system is non-ideal. The mean reconstruction errors and the associated standard deviations are shown in Fig. 5.

As expected, Fig. 5 shows that the mean 3D localization error of the real C-arm imaging system is always higher than the benchmark result obtained assuming an ideal system geometry. Note, however, that this difference can be rather small, e.g., only $0.16 \mathrm{~mm}$ when orthogonal views are used for triangulation. In this configuration, 


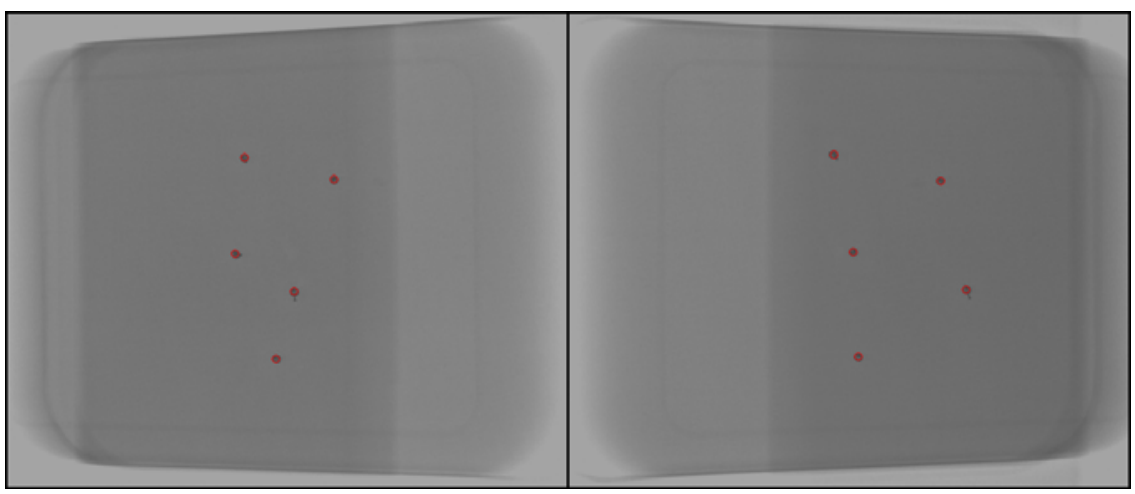

Figure 4. Pair of X-ray images of the phantom taken at $\alpha_{1}=30$ and $\alpha_{2}=-30$. The red circles indicate the $90 \%$ confidence interval around the ground truth. The ground truth in $2 \mathrm{D}$ is the forward projected 3D point position either using ideal projection matrices (simulation study) or online projection matrices (experimental evaluation).

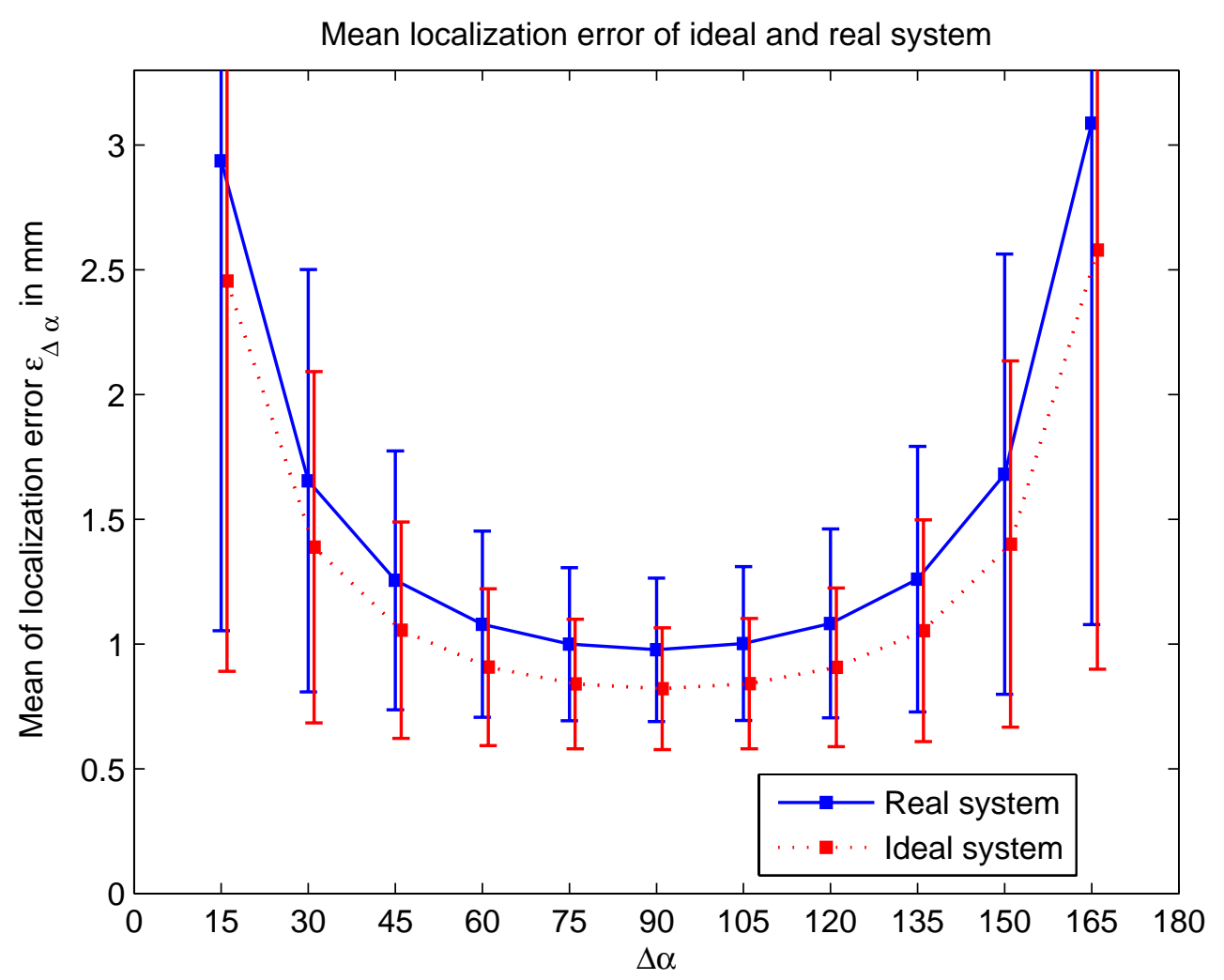

Figure 5. Mean reconstruction errors and associated standard deviations for an actual C-arm system (blue, solid) compared to results assuming an ideal projection geometry (red, dotted). (The small offset between the red and blue error bars is for a better graphical representation.)

the actual C-arm system performs almost as well as its idealized counterpart. Then the mean 3D error for an ideal system is $0.82 \mathrm{~mm}$, while the 3D localization error for a real system at this view setup amounts to 0.98 $\mathrm{mm}$. Reducing the angular difference between the two views to $30^{\circ}$, the mean $3 \mathrm{D}$ error for the ideal system and the actual C-arm device increases to about $1.39 \mathrm{~mm}$ and $1.65 \mathrm{~mm}$, respectively. The same effect is seen when increasing the angular view difference to $150^{\circ}$. Also note that not only are the reconstruction errors smallest for 
orthogonal views, the standard deviations are at their minimums as well. For the ideal (real) C-arm imaging device they are $0.24 \mathrm{~mm}(0.29 \mathrm{~mm})$ in an orthogonal view configuration and increase to $0.70 \mathrm{~mm}(0.85 \mathrm{~mm})$ for angular view differences of $30^{\circ}$ (or $150^{\circ}$ ).

\section{DISCUSSION AND CONCLUSIONS}

In this paper, we were interested in finding out how well a state-of-the-art C-arm system performs when used for stereotactic X-ray localization. To this end, we studied the 3D localization error between a reference position and its counterpart reconstructed by triangulation from two different $\mathrm{C}$-arm view directions. Gaussian noise with a standard deviation of $1.232 \mathrm{~mm}$ (4 detector pixels) was added to the 2D positions to simulate detection errors, e.g., when users do not click exactly at a particular 2D position or when automatic methods are somewhat off.

The results, obtained with a floor mounted AXIOM Artis dFA C-arm system (Siemens AG, Healthcare Sector, Forchheim, Germany), show that these systems have a reproducible, deterministic imaging geometry that facilitates 3D localization by triangulation with millimeter system accuracy. Since the reconstruction error for the real system turned out to be very close to what could be achieved with an ideal system, we also conclude that the online projection matrices for the real C-arm system were precise and reliable.

As expected the best reconstruction results were achieved using views that were orthogonal to each other, i.e., 90 degrees apart. This was true for the ideal device as well as for the real C-arm system. For orthogonal views, the results for an ideal system were $0.82 \mathrm{~mm} \pm 0.24 \mathrm{~mm}$ (mean \pm standard deviation), while the 3D localization error for a real system amounted to $0.98 \mathrm{~mm} \pm 0.29 \mathrm{~mm}$. Changing the angular difference between the two views to $30^{\circ}$, the $3 \mathrm{D}$ error for the ideal system and the actual C-arm device increased to $1.39 \mathrm{~mm} \pm 0.70 \mathrm{~mm}$ and 1.65 $\mathrm{mm} \pm 0.85 \mathrm{~mm}$, respectively. For a view angle difference of $150^{\circ}$, we get an average error of $1.40 \mathrm{~mm} \pm 0.73$ $\mathrm{mm}$ for the ideal projection geometry. The real C-arm system yielded a mean error of $1.68 \mathrm{~mm} \pm 0.88 \mathrm{~mm}$ at this configuration. Similar results have been reported for a stereotactic breast biopsy X-ray imaging setup. ${ }^{17,18}$ Not only were the reconstruction errors smallest for orthogonal views, the standard deviations were at their minimums as well. Put differently, uncertainties in 2D point detection matter less when views are orthogonal. This shows that it is beneficial to use orthogonal views for 3D object localization by triangulation.

A comparison with other navigations systems shows that 3D reconstruction from two X-ray views achieves similar accuracy. For example, the localization error for an electromagnetic system was reported to be $1.1 \mathrm{~mm}$ $( \pm 0.2 \mathrm{~mm})^{19}$. Optical tracking systems, on the other hand, can be characterized by a localization accuracy of between $0.26 \mathrm{~mm}$ and $2.86 \mathrm{~mm}$, depending on the system in use ${ }^{20}$.

\section{REFERENCES}

[1] Strobel, N., Meissner, O., Boese, J., Brunner, T., Heigl, B., Hoheisel, M., Lauritsch, G., Nagel, M., Pfister, M., Rührnschopf, E.-P., Scholz, B., Schreiber, B., Spahn, M., Zellerhoff, M., and Klingenbeck-Regn, K., "Imaging with Flat-Detector C-Arm Systems," in [Multislice CT (Medical Radiology / Diagnostic Imaging)], Reiser, M. F., Becker, C. R., Nikolaou, K., and Glazer, G., eds., 33-51, Springer.

[2] Penney, G. P., Registration of Tomographic Images to X-ray Projections for Use in Image Guide Interventions, PhD thesis, Computational Imaging Science Group, Division of Radiological Sciences and Medical Engineering, Guy's, King's and St. Thomas' School of Medicine, King's College London (December 1999).

[3] Prümmer, M., Han, J., and Hornegger, J., "2D-3D Non-rigid Registration using Iterative Reconstruction," in [Vision Modeling and Visualization], Greiner, G., Hornegger, J., Niemann, H., and Stamminger, M., eds., 187-194 (2005).

[4] Richter, G., Pfister, M., Struffert, T., Engelhorn, T., Dölken, M., and Dörfler, A., "Visualization of selfexpandable stents using 2D-3D coregistration of angiographic computed tomography data to facilitate stent assisted coil embolization of broad based intracranial aneurysms: in vitro feasibility study," in [42. Jahrestagung der Deutschen Gesellschaft für Neuroradiologie (DGNR 2007)], (2007).

[5] Galloway, R. L., "The Process And Development Of Image-Guided Procedures," Annual Review of Biomedical Engineering 3(1), 83-108 (2001). PMID: 11447058.

[6] Fajardo, L., Willison, K., and Pizzutiello, R., [A comprehensive approach to stereotactic breast biopsy], Blackwell Science Inc, Boston, MA (1996). 
[7] Schmidt, F., Catheter Localization in Coronary Biplane X-ray Imaging, Master's thesis, Friedrich-AlexanderUniversität Erlangen-Nürnberg, Germany (October 2005).

[8] Hartley, R. and Zisserman, A., [Multiple View Geometry in computer vision], Cambridge University Press.

[9] Rougee, A., Picard, C. L., Trousset, Y. L., and Ponchut, C., "Geometrical calibration for 3D x-ray imaging," Medical Imaging 1993: Image Capture, Formatting, and Display 1897(1), 161-169, SPIE (1993).

[10] Gorges, S., Kerrien, E., Berger, M. O., Trousset, Y., Pescatore, J., Anxionnat, R., and Picard, L., "Model of a Vascular C-Arm for 3D Augmented Fluoroscopy in Interventional Radiology," Medical Image Computing and Computer-Assisted Intervention MICCAI 2005, 214-222, MICCAI (2005).

[11] Golub, G. and Kahan, W., "Calculating The Singular Values And Pseudo-Inverse Of A Matrix," J. SIAM Numer. Anal. 2(2), 205-224 (1965).

[12] Noo, F., Hoppe, S., Dennerlein, F., Lauritsch, G., and Hornegger, J., "A new scheme for view-dependent data differentiation in fan-beam and cone-beam computed tomography," Physics In Medicine And Biology 52(17), 5393-5414 (2007).

[13] Scherl, H., Keck, B., Kowarschik, M., and Hornegger, J., "Fast GPU-Based CT Reconstruction using the Common Unified Device Architecture (CUDA)," in [Nuclear Science Symposium, Medical Imaging Conference 2007], Frey, E. C., ed., 4464-4466 (2007).

[14] Scherl, H., Koerner, M., Hofmann, H., Eckert, W., Kowarschik, M., and Hornegger, J., "Implementation of the FDK Algorithm for Cone-Beam CT on the Cell Broadband Engine Architecture," in [Proceedings of SPIE Medical Imaging 2007: Physics of Medical Imaging], Hsieh, J. and Flynn, M., eds., 6510, 651058 (2007).

[15] Zellerhoff, M., Scholz, B., Ruehrnschopf, E.-P., and Brunner, T., "Low contrast 3D reconstruction from C-arm data," in [Proc. of SPIE Medical Imaging 2005: Physics of Medical Imaging], Flynn, M. J., ed., Medical Imaging 2005: Physics of Medical Imaging 5745(1), 646-655, SPIE (2005).

[16] Fahrig, R. and Holdsworth, D. W., "Three-dimensional computed tomographic reconstruction using a C-arm mounted XRII: Image-based correction of gantry motion nonidealities," Medical Physics 27, 30-38 (January 2000).

[17] Jiang, H., Liu, H., Wang, G., Chen, W., and Fajardo, L. L., "A localization algorithm and error analysis for stereo x-ray image guidance," Medical Physics 27(5), 885-893 (2000).

[18] Jiang, H., Chen, W. R., Wang, G., and Liu, H., "Localization error analysis for stereo X-ray image guidance with probability method," Medical Engineering and Physics 23, 573-581 (2001).

[19] Nagel, M., Hoheisel, M., Petzold, R., Kalender, W. A., and Krause, U. H. W., "Needle and catheter navigation using electromagnetic tracking for computer-assisted C-arm CT interventions," in [Proceedings of SPIE Medical Imaging 200\%: Visualization and Image-Guided Procedures], Cleary, K. R. and Miga, M. I., eds., Proc. of SPIE 6509, 65090J-1-65090J-9 (2007).

[20] West, J. B. and Maurer, C. R., "Designing Optically Tracked Instruments for Image-Guided Surgery," IEEE Transactions on Medical Imaging 23(5), 533-545 (2004). 